\title{
REGISTRO DE LOS CARTÓGRAFOS MEDIEVALES ACTIVOS EN EL PUERTO DE MALLORCA
}

GABRIEL LLOMPART

Universitat de les Illes Balears

Un buen conocedor de la cartografía medieval ha escrito poco ha estas palabras en el primer volumen de la Historia de la Cartografía de J. B. Harley y David Woodward a propósito del comercio de las cartas: "Nuestra general ignorancia acerca de las personas de los cartógrafos va al unísono con la limitadísima información de que disponemos sobre sus clientes"1.

Es verdad que contamos cada vez con más noticias acerca de la cartografía medieval, pero también es verdad que entre nosotros tardan mucho en entrar en la bibliografía nacional o internacional.

Para no citar más que un caso chirriante, aquí están las dos monografías dedicadas por Jaume Riera i Sans a los cartógrafos Cresques Abraham (1325-1387) ${ }^{2}$ y Jafuda Cresques (1360-1407) ${ }^{3}$, escritas en torno al centenario del atlas catalán -o mallorquín o como se le quiera llamarde 1375. Apenas han tenido eco en las nuevas ediciones extrangeras del Atlas y ni siquiera figuran en la bibliografía de la Historia de la Cartografía que citábamos más arriba.

'T. CAMPBELL, Portolan charts from the late thirteenth century to 1500 in J.B. HARLEY, D. WOORWARD, The History of cartography, vol. I, Chicago-London 1987, p. 435.

${ }^{2} \mathrm{~J}$. RIERA SANS, Cresques Abraham, jueu de Mallorca, mestre de mapamundis $i$ de bruxoles in L'Atlas català de Cresques Abraham, Barcelona, Diàfora, 1975, pp. 14-22.

${ }^{3}$ J. RiERA SANS, Jafuda Cresques, jueu de Mallorca "Randa" 3 (1977), pp. 51-66.

"Anuario de Estudios Medievales", 27 (1997) 
La queja de Toni Campbell afecta, pues, a nuestro modo de ver no sólo a los cartógrafos y a los clientes sino también a los investigadores.

El estudio que ofrecemos -dando de lado a las jeremiadaspretende hacer alguna luz sobre uno de los centros cartográficos importantes del Mediterráneo medieval. Se trata del puerto de la Ciutat de Mallorques (hoy Palma de Mallorca y en la Edad Media, en la documentación latina, Civitas Maioricarum; locativo geográfico: Maioricis).

Hasta ahora en los estudios de Historia de la Cartografía se había centrado la atención sobre las piezas cartográficas y, a través de ellas, se sacaba la información sobre los cartógrafos, sus autores. Ciertamente, también se habían aprovechado los documentos procedentes de los archivos, recogidos por los investigadores mallorquines adscritos a la veterana Societat Arqueológica Lul-liana. Luego el mencionado hebraista J. Riera Sans escudriñó a fondo el Archivo de la Corona de Aragón para hacerse una idea cabal de las figuras de Cresques Abraham y Jafudà Cresques.

Con motivo de preparar mi tesis sobre La pintura medieval mallorquina, su entorno cultural y su iconografía comencé a recoger datos sobre la cultura visual en toda su amplitud: pintura, miniatura, bordado, platería y cartografía. Así pude hacerme cargo de la situación real de la investigación en el campo cartográfico y los dibujantes del puerto y para el puerto (navegantes, comerciantes) dedicándole un apartado ${ }^{4}$. A partir de aquí J. N. Hillgarth, al publicar su monumental catálogo Readers and Books in Majorca 1229-1550, ya recogió en sus índices generales los conceptos de Map-makers, Maps and Navigational charts utilizando fundamentalmente fondos de protocolos notariales ${ }^{5}$. Aquí radican las informaciones - precisamente en las notarías- que en un inmediato futuro puede que hagan circular savia y nueva vida sobre los resecos pergaminos y los esqueléticos datos con que contamos a propósito de los dibujantes de los mapas medievales de Mallorca. Y podemos añadir, del resto del Mediterráneo, cuando se ahonde también en sus protocolos notariales...

En las nuevas búsquedas realizadas estos años han aparecido noticias, ciertamente escuetas, a menudo indirectas, sobre los cartógrafos comenzando

\footnotetext{
${ }^{4} \mathrm{G}$ LLOMPART, La pintura medieval mallorquina, su entorno cultural y su iconografía, 4 vols. Palma, Luis Ripoll ed., 1977-1980, especialmente pp. 172-181.

${ }^{5}$ Jocelyn N. HILLGARTH, Readers and Books in Majorca 1229 - 1550, 2 vols, Paris, Editions du Centre Nationale de la Recherche Scientifique 1991, especialmente vol. II, Index I, pp. 891 y 896.
} 
en el siglo XIV y acabando en el siglo XVII, cuando los dibujantes a pluma del Sur de Europa fueron superados por la técnica tipográfica de las imprentas de los Paises Bajos del Norte.

Es conocido y reconocido el mérito del elenco de mapas $-\mathrm{y}$, por ende, de cartógrafos medievales de Mallorca- que recogieron los expertos Julio Rey Pastor y Ernestò García Camarero en el libro La cartografía mallorquina, aparecido en Madrid en $1960^{6}$. El trabajo en perspectiva ha de ser el componer las biografías de los respectivos cartógrafos en los archivos de los puertos en los que desempeñaron su oficio y consiguieron un mercado para su propio beneficio. Este trabajo tiene cierto parecido con el de zurcido de redes de los pescadores de bajura.

Ofrecemos, por tanto, en estas páginas un manojo de noticias de índole variada destinadas a mejorar el elenco formado por Rey Pastor y García Camarero con los autores de la Cartografía Mallorquina. Si es el caso de advertir sobre algún dato aparecido en la bibliografía local de los últimos tiempos que enriquezca la ficha de alguno de ellos, se procurará hacerlo. Hay que advertir que, en algunos casos, hasta el presente ni siquiera se ha fijado la nomenclatura de los sujetos. A estas alturas no se puede consentir que estos hombres de la mar ignorati damnentur.

Después del hallazgo del documento de reconocimiento de aprendizaje, hace unos años, del judío Samuel Corcós en vez del maestro Jafuda Cresques, parece estar probado que la llamada Escuela Cartográfica de Mallorca se reduce a la relación "aprendiz", "oficial", "maestro", en forma rudimentaria ${ }^{7}$. En bloque, la formulación tradicional no sería más que la secuencia de parejas "maestro-menestral" en el ámbito del dibujo especializado en la geografía marina, teniendo por fondo el puerto de Mallorca (que así se llamaba sencillamente antaño el que denominamos actualmente de Palma de Mallorca) por una duración de varios siglos, desde la apertura de la ruta atlántica (siglo XIV) hasta el perfeccionamiento de la imprenta (siglo $\mathrm{XVII})^{8}$. Este perfeccionamiento dejó sin trabajo a la copia de mapas a

${ }^{6}$ J. Rey PASTOR, E. GarCía CAMARERo, La cartografía mallorquina, Madrid, CSIC, 1960.

${ }^{7}$ G. Llompart, J. RIERA, Jafudà Cresques $i$ Samuel Corcós. Mes documents sobre els jueus pintors de cartes de navegar (Mallorca, segle XIV), "BSAL", 40 (1984), pp. 341-350, especialmente p. 349.

${ }^{8}$ En efecto Pere Joan Prunès "menestral de cartes de navegar" reconoce haber recibido en testamento de su padre el maestro Vicenc Prunès la mitad de sus patrones de cartas de navegar (1625). Cfr. G. LlOMPART, Identificación del Maestro de las Predelas y encuadre de otros mas, "Estudis Baleàrics", 29-30 (1989), pp. 45-52 (p. 49). 
pluma, sobre plantillas y su venta y despacho local, en lo que se había convertido ya la cartografía tradicional. El que el puerto de Mallorca alcanzara la categoría de aprovisionador del mercado de mapas se debió unicamente a su situación geográfica. Esta posición privilegiada aparece acuñada como una tradición secular en el momento de la conquista de Mallorca en 1229, en el discurso de Pere Martell tenido ante Jaime I el Conquistador en Tarragona. Y sobre todo en el comentario que de él hace el cronista dominico fra Pere Marsili O.P. (1314), el cual, conocedor directo de la isla, dice "los homens d'aquela art (de marineria) aquella yla apeylan cap de creus com d'aquela a cascunas parts navegar pus cuvinetment es vist"9. Quien tenga presente que, en vida de este fraile inquieto y viajero, apareció la red de rumbos llamada marteloio en las cartas marinas y que nos encontramos en las fechas en que se dibujaron las primeras cartas conservadas del genovés Pietro Vescomte $\left(1311,1313,1318,1320^{10}\right)$ no le extrañará el excurso con que cierra su Crónica dedicada a explicar el nombre de los vientos usados por los navegantes (nautxers, nautxerants). Cualquiera diría que él entonces recordaba una explicación recibida en alta mar, en la popa de una galera, cabe la caseta de los instrumentos de navegación. "Cap de creus" es lo mismo que el castellano "crucero" o el francés "carrefour" "1.

La relación de los cartógrafos recogidos en esta documentación archivística del puerto de Mallorca, por orden cronológico, es la siguiente: Angelinus Dulcieti, Civis Majoricarum, docs. 1, 2, 3 (1344).

Guillermus Canterel, doc. 4 (1353).

Guillermus Canterelli, bruxulerius et cives Major. docs. 5 (1354), doc. 6 (1362).

Guilermus Solerii $(\dagger)$, doc. 7 (1402) buxolerius, mestre de cartes de navegar.

Gabriel Vallsecha, doc. 8 (1437), 9 (1438), 10 (1443).

Gabriel Soler, magister cartarum navigandi, buxoler, doc. 11 (1446), doc. 12 (1463), doc. 13 (1467).

\footnotetext{
${ }^{9}$ J.M. Quadrado, Historia de la Conquista de Mallorca, Palma 1850, pp. 21-22.

${ }^{10} \mathrm{~T}$. CAMPBell, Portolan charts, cit. en nota 1, p. 408.

"Véase el sugestivo apunte de la caseta o "tabernacle" en A. GARCIA SANZ y N. COLL JULIÀ, Galeres mercants catalanes dels segles XIV y XV, Barcelona, Fundació Noguera, 1994, p. 194.
} 
Antonius Bramona, buxolerius, nationis sarracenorum, doc. 14 (1451)

Rafael Monells, buxoler, doc. 15 (1454).

Pere Rossell, buxolerius, civis Maior., doc. 16 (1463), doc. 17 (1474).

Jaume Bertrán, buxolerius $(\dagger)$ doc. 18 (1500).

Joan Salvat, illuminator, buxoler, doc. 19 (1513), doc. 20 (1520), doc. 29 (1563).

Bartomeu Olives senior, pintor, docs. 21, 22, 23, (1498, 1506, 1514).

Bartolomeus Olives iunior, pictor, illuminator cartarum navigandi docs. 25, 26, 27, 28, 29.

Vincentius Prunes, cosmographus, doc. 30 (1609).

A ello podemos juntar las referencias que hemos recogido en otros trabajos anteriores:

Samuel Corcós, judío, Macià de Viladesters (de converso)

buxoler, documentado en Mallorca en los años 1370, 1383, $1390,1401,1404,1409,1421^{12}$.

Cresques Abraham ${ }^{13}$ mestre de cartes de navegar.

Jafuda Cresques ${ }^{14}$ (de converso, Jaume Ribes) id.

Astruc Cresques ${ }^{15}$, qui fa mapamundis.

Gabriel de Vallseca, buxoler, documentado en Mallorca entre 1439 y $1467(\dagger)^{16}$.

Rafael Monells, pintor, buxoler, documentado en 1451, $1468(\dagger)^{17}$

Antonius Piris, buxolerius, civis Maioric. $1463^{18}$.

Gabriel Soler, buxoler 1449 a $1474^{19}$.

${ }^{12}$ G. LLOMPART, La cartografía mallorquina del siglo XV. Nuevos hitos y rutas, "BSAL" 34 (1975), pp. 438-465, en pp. 441-442.

${ }^{13}$ Véase la bibliografía en RIERA SANS, Cresques Abraham, cit. en nota 2.

${ }^{14}$ Véase Riera SANS, Jafuda Cresques, cit. en nota 3, con la bibliografía al día.

${ }^{15} \mathrm{G}$. LlOMPART, Documentos sueltos sobre judios y conversos de Mallorca (siglos XIV y $X V$ ), en "Fontes rerum Balearium", 2 (Palma 1978), núm. 1, p. 187.

${ }^{16} \mathrm{G}$. LLOMPART, La cartografía cit., especialmente pp. 442-446.

${ }^{17}$ G. LLOMPART, La cartografía cit., p. 447.

${ }^{18}$ G. LLOMPART, La cartografía cit., p. 444.

${ }^{19}$ G. LlOMPART, La cartografía cit., p. 447. 
Pere Rossell, buxoler, documentado entre 1463 y $1474^{20}$.

Jaume Bertrán, buxoler, documentado en Mallorca entre 1478 y $14902^{21}$.

Rafel Loret, mestre de cartes de navegar, $1436^{22}$.

Ioannes Salvat, buxolerius, aurifaber, $1523^{23}$.

Bartomeu Oliva, pintor, 1504, $1512^{24}$.

$\mathrm{Y}$ en otro trabajo en prensa figuran más datos referentes: a Gabriel Vallseca (docs. 33-36), entre 1451 y 1465, a Pere Rossell (doc. 22) de 1474 y a Bartomeu Olives (doc. 19) de $1492^{25}$.

Las últimas perquisiciones acerca del clan judío llamado impropiamente de los Cresques ha dado material para unas notas sobre su Sitz im Leben en el ghetto de Mallorca pero llama la atención el que no hagan alusiones a su profesión de miniaturistas especialistas en mapas ${ }^{26}$.

El primer cartógrafo que firma cartas geográficas con nombre y apellido propios y la referencia topográfica de la Ciudad de Mallorca lo hace usando esta cartela: Hoc opus fecit Angelino Dulcert, anno MCCXXXVIIII, de mense augusti in civitate Maioricarum (Paris, Bibliothèque Nationale. Rés. Ge. B 696).

A partir de esta referencia, ha sido posible dar efectivamente con la presencia del personaje en cuestión en unas casas del casco viejo gótico de la Ciudad de Mallorca (docs. 1-3). El mencionado Angelinus Dulcieti, civis Maioricarum, tenía su domicilio en la parroquia de San Nicolás, en la calle den Comes, lindante con el albergue de Tomás Assati y con la "carniceria d'avall". En otras palabras, habitaba en la primera fila de manzanas flanqueando el torrente de la Riera, que entonces cruzaba la población, y tenía un puente de distribución a la altura de la actual plaza del Rey Juan Carlos.

${ }^{20} \mathrm{G}$. LLOMPART, La cartografía cit., p. 447-448.

${ }^{21} \mathrm{G}$. LLOMPART, La cartografía cit., p. 448.

${ }^{22} \mathrm{G}$. LLOMPART, La cartografía cit., p. 448.

${ }^{23}$ G. LLOMPART, La cartografía cit., p. 448.

${ }^{24} \mathrm{G}$. LLOMPART, La cartografía cit., p. 448.

${ }^{25}$ M. BARCEló, G. LlOMPART, Quaranta dades d'art medieval mallorquí, "BSAL", 54 (1998) pp. 85-108.

${ }^{26} \mathrm{G}$. LlOMPART, El testamento del cartógrafo Cresques Abraham y otros documentos familiares, "Estudis Baleàrics", en prensa. 
Es cierto que los documentos no aportan la calificación profesional del sujeto - es decir no lo llaman "buxoler" que era la designación coetánea. Pero, en cambio, sí nos lo presentan como Civis Maioricarum. Estos datos resultan insuficientes para garantizarnos el origen del maestro cartógrafo: genovés o mallorquín.

De todos modos, he de subrayar ahora que las manzanas de primera fila de la ciudad, durante el período conocido como el Reino privativo de Mallorca, tienen un importante porcentual de pobladores de apellidos italianos, a partir de los molinos vecinos al Hort del Rei, siguiendo por la Llotja dels Genovesos (junto a la central de Telégrafos) hasta las edificaciones levantadas sobre al abandonado convento de Santa Margarita, antes de llegar al actual Teatro Principal. Sobre el convento hubo enseguida una colonia de judíos e italianos.

Un examen atento de la serie "Escrivania de Cartes Reials", del Archivo del Reino de Mallorca, me ha permitido llegar a la conclusión de que así como el nombre "Angelina" en la primera mitad del siglo XIV es usual entre la población femenina, en cambio el de "Angelino" comparece pocas veces y lo llevan siempre sujetos italianos.

Vayan unos ejemplos para ponderarlos: Angelinus Andree, hijo de Augustinus Andree, civis Maioricarum, firma un contrato de trabajo con el patrón italiano Simon Iuliani, como pescador de jábega, en $1340^{27}$; Angelinus Pisani, civis Maioricarum, circa 20 años de edad, hijo de Petrus Pisani y su viuda Caterina Caldura, venden casas en el viejo convento de Santa Margarita a G. Scot, genovés, en 1319 $9^{28}$; Angelino de la Macdalena, ianuensis, civis Maioricarum, adquiere una casa en el mismo convento a Serena, cuñada de Guillem Vadell senior, notario y autor de la Summa Vadellina, en 131429; Angeli Scot, ciertamente italiano, probablemente genovés ${ }^{30}$.

De momento y hasta que se haya realizado una prospección en la onomástica de la República de Génova, que hasta 1359 se alargaba hasta Niza, no me parece útil dar los resultados acerca de la misma prospección

\footnotetext{
${ }^{27}$ ARM. ECR-364, f. $177 \mathrm{v}$.

${ }^{28}$ ARM. ECR-358, f. 302.

${ }^{29}$ ARM. ECR-358, f. 264v. En 1336 figura su domicilio en el padrón de la parroquia de San Nicolás, aunque se halla ausente. J. MIRALles MONTSERRAT, Corpus d'antropònims mallorquins del segle XIV, Barcelona 1997, p. 203.

${ }^{30}$ Ya fallecido en 1336. J. MiRalles, Corpus de toponimia, cit., p. 227.
} 
verificada a propósito del apellido "Dulcert" en los archivos de protocolos de Mallorca, atendida su complejidad. De todos modos, no quiero dejar de dar mi opinión acerca de la nacionalidad del primer cartógrafo de nombre conocido que trabaja en Mallorca. Debía ser genovés aunque poseía como la mayoría de sus connacionales aquí también la nacionalidad mallorquina. Así que frente al 2000 huelgan las discusiones patrióticas y se agradecen las búsquedas de antroponimia.

Los documentos 4,5 y 6 se refieren a un cartógrafo hasta ahora desconocido. Un tal Guillermus Canterel, Canterele o Canterelli, civis Maioricarum, que adquiere una casa en el mismo márgen izquierdo de la Riera, cercana al llamado Hort del Rei. Está datado en los años 1353, 1354 y 1362. Es sin duda el mismo ciudadano que comparece en un censo de mitad del siglo XIV, en la misma parroquia de San Nicolás, y que ha dejado un tanto perplejo al trascribirlo al Dr. Joan Miralles: En Guillem Quanter(e)s [?] ... Habrá de leerse probablemente Canterelles, que es la forma más usual entre nosotros. Su nacionalidad original resta, me parece, incierta ${ }^{31}$.

El documento 7 nos presenta al conocido cartógrafo Guillem Soler, aunque ya fallecido. Es la primera vez que aparece una noticia acerca de este hombre, aparte de la firma de sus mapas. Rey Pastor y García Camarero lo fechaban por las mencionadas firmas de sus mapas en París y en Florencia en 1380-1385. El documento en cuestión se refiere directamente a una hija suya llamada Margarida, casada con un cierto Esteban Loret. La fecha es de 1402 y la pieza se refiere a unas casas situadas en la calle de Bonaire, en la Parroquia de Santa Creu.

El interés de estas líneas es doble. Por la razón de que poseíamos algunas referencias a un cartógrafo de la primera mitad del siglo XV (1436) apellidado Rafel Loret, sin obra conocida. Dada la posible relación de parentesco entre Solers y Lorets se apunta asimismo una lógica filiación de cartas ... por descubrir ${ }^{32}$.

Pasemos ahora a Gabriel Vallseca. Los documentos 8 a 10 se refieren al converso catalán, establecido en el puerto de Mallorca y cuyo testamento del 1467 publicó hace unos años F. Sevillano. Precisamente el núm. 9 es la concesión oficial de la ciudadanía mallorquina con sus derechos

\footnotetext{
${ }^{31} \mathrm{~J}$. MIRALLES, Corpus d'antropònims mallorquins, cit., p. 501.

${ }^{32}$ Gabriel LLABRÉs, Galería de artistas mallorquines, "BSAL", 18 (1920-1921), pp. 198 199. G. LlOMPART, La cartografía mallorquina del siglo XV. Nuevos hitos y rutas, "BSAL", 27 (1973-75), pp. 438-465, especialmente p. 465.
} 
correspondientes a nuestro hombre calificado como "mestre de cartes de navegar" (1438).

Los restantes se refieren a negocios de siervos (núm. 89) y a adquisición de inmuebles (núm. 10) propiedad de ciudadanos florentinos de la familia di Manzoli (1443). Queda por tanto documentada su posible relación comercial profesional con Italia.

Con ello se amplian algo las noticias biográficas de este cartógrafo cuya relación dí hace años ${ }^{33}$.

El cartógrafo Gabriel Soler, desconocido por Rey Pastor y García Camarero, calificado tanto de buxoler como de magister chartarum naviguandi, figura en los documentos 11 a 13 entre los años 1446 y 1467. Le conocíamos ya por un censo de la parroquia de Santa Cruz anterior a $1467^{34}$.

Un brujulero inesperado resulta ser cierto Antoni Bramona, de nación de sarracenos, al que alquila la familia des Brull parte de su casa situada en el Born de Palma de Mallorca (doc. 14). Efectivamente la población se muestra reacia en el siglo XV a aceptar que antiguos esclavos que pertenecieron a gremios colindantes con la navegación fueran liberados y retornaran a sus tierras por temor a que dirigieran después razzias piráticas por la costa mallorquina. Estamos en 1451 aunque desconocemos el contexto biográfico de este profesional norteafricano.

El documento 15 se refiere a Rafel Monells, un pintor $-\mathrm{y}$ antes brujulero- del que conocíamos el inventario post mortem (1468) y al que encontramos en 1454 en plena quiebra económica, después de haberse desprendido de la casa que antaño comprara al pintor Joan Marçol. Es el único caso conocido de pintor de mapas (1415-1468) que se pasará a pintor de retablería. No se ha conservado ninguna obra suya, aunque se hallaron en su taller tanto cartas como retablillos, sin especificar. Al final de su vida está relacionado con el taller del pintor Nicolau Amat (1440-1497) ${ }^{35}$.

Los documentos 16 y 17 se refieren a Pere Rossell, dando cuenta de compras de textiles y grasas. Le muestran en su casa de la parroquia de

\footnotetext{
${ }^{33} \mathrm{G}$. LLOMPART, La cartografía mallorquina, cit., pp. 442-446. Y de nuevo, M. BARCELÓ, G. LLOMPART, Quaranta dades d'art medieval mallorquí, "BSAL", 54 (1998), pp. 85-108, concretamente en pp. 103-105.

${ }^{34}$ G. LlOMPART, La pintura medieval mallorquina, vol. 4, Palma 1980, doc. núm. 20.

${ }^{35} \mathrm{G}$. LlOMPART, La cartografía mallorquina cit. pp. 447; G. LLOMPART, La pintura medieval mallorquina, vol. 4, Palma 1978, docs. 315-319.
} 
Santa Creu, vecino de las atarazanas. Rey Pastor y García Camarero, que recensionan una quincena de cartas naúticas, firmadas en Mallorca la mitad de ellas, lo colocan entre las fechas de 1446 y 1489 . Ahora lo encontramos en el puerto en los años 1463 y $1474^{36}$.

También Jaume Bertrán, documentado entre 1456 y 1490, al que Rey Pastor y García Camarero reconocen cartas firmadas en Mallorca en 1482 y 1489, tenía casa abierta en la parroquia de Santa Cruz y no sólo casa sino familia que aparenta tener algunas dificultades a propósito de la herencia paterna en 1500. Podemos pues en virtud de nuestro documento 18 fijar la fecha de su muerte en $1500^{37}$.

Joan Salvat, presente en los documentos 19 y 20, figura como illuminator en 1513, como buxoler en 1520 y como buxolerius aurifaber en 1523. En sus cartas de la Armeebibliothek de Muenchen (n. 31, 1) y de la Biblioteca Pública de Toledo (ms. 530) firma Salvat de Pilestrina sencillamente. Es una figura menor pero habrá que aclarar su filiación: de cierto trabaja en Mallorca entre 1511 y 1520 , como aquí vemos, aunque la carta conservada en la Biblioteca Pública de Toledo va fechada en Mallorca en $1533^{38}$

Los documentos 21 a 29 ayudan a situar una larga familia o clan de dibujantes de mapas: los Olives, cuya labor catalogó el manual de Rey Pastor y García Camarero dedicándoles 44 páginas en las que resigue sus vidas y estancias en Mallorca, Nápoles, Mesina, Marsella y Livorno. Total: doscientos años de empresa. Las referencias que aportamos se refieren solamente al arranque de la familia.

En los "Capítulos de la Cofradía de pintores", fundada en Mallorca en 1512 ocupan los lugares 4 y 5 del elenco de artistas fundadores Bartomeu Oliva (sic) y Joan Salvat, a quien ya conocemos ${ }^{39}$. Ambos eran amigos, según se desprende de los papeles. Es probable que al venir a Mallorca a pintar a la cartuja de Valldemossa Manuel Ferrando, procedente de Italia, se amparara en el taller de Bartomeu Oliva. Así se explicaría su colaboración de entonces. Sin embargo el taller de pintura de la calle del Mar, la entrada

\footnotetext{
${ }^{36} \mathrm{G}$. LLOMPART, La cartografía mallorquina cit., pp. 447-448.

${ }^{37}$ Rey Pastor, García CamarerP, La cartografía mallorquina cit., pp. 82-83. G. LLOMPART, La cartografía mallorquina cit., p. 448.

${ }^{38} \mathrm{~V}$. ROSSELLÓ VERGER, Els italianismes de les cartes portolanes mallorquines in "Estudis de lengua y literatura en honor de Joan Veny", vol. I (1997) pp. 39-62, en p. 59.

${ }^{39} \mathrm{G}$. LlOMPART, La pintura medieval, cit., vol. 4 , doc. 24.
} 
en la ciudad, con todos sus almacenes y tiendas de mercaderes, hostales de la marina, notarios y pesajes reales, se hubo de convertir en un taller de cartografía. Mestre Bartomeu Oliva muere en 1514 (doc. 23). Había trabajado para la Catedral, como nos consta pero también había tomado en 1498 un ayudante portugués Alvaro de Vieira, de Lisboa. No sabemos mucho de esto, sólo que no era un aprendiz y que fue avalado por negociantes genoveses.

El doc. 25 muestra a Bartomeu Olives iunior que adquiere una importante cantidad de pergaminos. El notario le llama simplemente pictor $(1532)^{40}$. El documento 28 cambia de trayectoria. Trae como protagonista a Bartomeu Olives, pero no es el que llamamos iunior, casado con Francina, sino otro del mismo nombre y presumiblemente hijo suyo, casado en 1546 con Margarita y que se cualifica como illuminator cartarum navigandi. Mientras tanto, la viuda Francina Oliva vive aun en 1563 y, a su lado, como testigo comparece Joan Salvat aurifaber a quien conocemos también y que en 1523 se llamaba de consuno buxolerius aurifaber ${ }^{41}$.

El último documento que presentamos en este repertorio de cierto peso específico es el 30 que se planta en 1609 y es el testamento de Vicenç Prunés que califica de cosmographus. Ya no vive en el barrio marinero sino en la ciudad alta, en la parroquia de Santa Eulalia, aunque sus testigos son gente de mar. Vicenç Prunés había sido identificado por Gonzalo de Reparaz como autor de un atlas del Museo Marítimo de Barcelona datado en 1600.

Este autor ha de ser el heredero de la obra de Mateu Prunés, catalogado por Rey Pastor y García Camarero y que consta de trece cartas naúticas y un atlas fechadas en Mallorca entre los años de 1552 y 1594.

Pero hay más. Porque quince años después de la muerte de Vicenç, se redacta un acta notarial, en virtud del cual su hijo Pere Joan Prunés, que se define como menestral de cartes de navegar, se reconoce heredero de la mitad de los modelos de sus mapas, dando a entender así que su hermana María, en el momento de su boda, había de recibir en concepto de dote la restante mitad.

De esta forma se rubrica el final de lo que hasta ahora se llama misteriosamente la escuela cartográfica mallorquina con la afirmación inicial del que hace unos años pudimos demostrar que fue el primer anillo de

\footnotetext{
${ }^{40} \mathrm{G}$. LLOMPART, La pintura medieval, cit. vol. 4 , doc. 415 .
}

${ }^{41}$ G. LLOMPART, La cartografía mallorquina cit. p. 465. 
aprendizaje: el reconocimiento de parte de Samuel Corcós de haber aprendido faciendi buxolas et illuminandi de parte de Jafuda Cresques magister de cartes de navegar (1390). Era la típica relación medieval entre mestre y menestral ${ }^{42}$.

\footnotetext{
${ }^{42}$ Algunas de estas notas fueron adelantadas en G. LlomparT, J.M. Palou, Apunts iconogràfics des del Port de Mallorca in Olga López (coord.) "Cartografia mallorquina", Barcelona, Museu Marítim, Diputación de Barcelona, 1995, pp. 71-87.
} 


\section{DOCUMENTOS}

1344, agosto, 4.

Angelí Dulcert tiene casa propia en la parroquia de San Nicolás, de la "Ciutat de Mallorques".

ARM. ECR 366, 313 v.

Item vendo ego, dictus Guillermus Castany, vobis, dicto Petro Rossinyoli, XII solidos censuales, quos facit et facere tenetur et amodo vobis et vestris faciat et facere teneatur, Angelinus Dulcieti, singulis annis, perpetuo, in festo Natalis Domini, supra quodam hospitio suo, quod est in parrochia Sancti Nicholay, in vico quod dicitur den Comes, in portione quae fuit Domini Nunii Sancii, quae nunc est domini regis, per quem tenetur ad VI denarios de laudimio, prout in cartis.

Et affrontatur dictum hospitium, ex una parte, in via publica, et, ex alia parte, in hospitio Thome Assati, et, ex alia parte, cum alio hospitio dicti Angelini, et, ex alia parte, cum carneceria inferiori.

Predictos itaque XII solidos censuales, cum omni iure et titulo, vobis vendo pretio videlicet sex 11., XIIII ss. et duorum denariorum etc., computato Regale pro viginti solidos, encameratas quas habui [...] Ad haec, ego, Astasia, uxor dicti G. Castany, laudans.

1344, agosto, 4 .

Angeli Dulcert paga un censo anual por su albergue de la calle "den Comes", antecitado. 
ARM. ECR 371, ff. 48 v-49 v.

Ego Guillermus Castany, civis Maioricarum, gratis et ex certa scientia, vendo et titulo venditionis cedo vobis, Petro Rossinyolli, mercatori, civi Maioricarum, presenti ementique legitime et sine fraude et vestris successoribus, duodecim solidos monete perpetue regalium Maioricarum minutorum censuales, quos michi facit et facere tenetur et amodo titulo et iure huiusmodi emptionis vestre vobis et vestris faciat et facere teneatur, annis singulis, perpetuo, in festo Nativitatis Domini, Angelinus Dulcieti, civis Maioricarum, pro quodam hospitio suo, quod est in parrochia Sancti Nicholay, in vico qui dicitur den Comes, in portione videlicet quae quondam fuit nobilis domini Nunonis Sancii, quae nunc est domini regis, per quem tenetur prout in instrumentis inde factis continetur.

Et affrontatur dictum hospitium, ex una parte, cum via publica, et, ex alia parte, cum hospitio Thome Assati, et, ex alia parte, cum alio hospitio dicti Angelini, et, ex alia parte, cum carneceria inferiori [...]

Actum est hoc in civitate Maioricarum, pridie nonis augusti, anno Domini MCCCXLIIII ${ }^{\circ}$.

Signum mei, Guillermi Castany. Signum Astasie, eius uxor. Signum Francisci Castany eorum filius predictorum, qui haec laudamus et firmamus.

1345, enero, 7.

Angelí Dolcert comparece como testigo en un contrato inmobiliario en la "Ciutat de Mallorques".

ARM, ECR 371, f, 102 v - 103 v.

VII idus januarii anno Domini MCCCXLIIII ${ }^{\circ}$

Ego Benencasa, uxor Daniel Tanis, civis Maioricarum quondam, gratis consulte et ex certa scientia, per me et meos presentes et futuros, do, stabilio et in enphiteusim perpetuam concedo ad bene videlicet operandum, meliorandum et non in aliquo deteriorandum, vobis, Guillermo Ros apothechario, civi Maioricarum presenti et vestris et quibus volueritis, perpetuo, quasdam domos meas sive hospitium quae sunt intus civitatem Maioricarum, satis prope pontem inferiorem, in portione videlicet quae quondam fuit nobilis Nunionis Sancii, quae nunc est domini regis Maioricarum, et per ipsum dominum regem tenentur ad censum quinque solidorum monete perpetue regalium Maioricarum minutorum, ipsi et suis dandorum 
et solvendorum, annis singulis, in festo Pasche Domini, quem censum ego et mei sive vestri et vestrorum dominio et missione ex censu infrascripto solvere tereamur.

Et affrontantur dicte domus, ex una parte, cum domibus Francisci Oliverii curritoris, et, ex alia parte, cum domibus Bartolomei Segalas, et, ex aliis duabus partibus, cum viis publicis.

Predictas itaque domos [...] do, stabilio et in enphiteusim perpetuam concedo, sub tali tamen pacto et conditione quod pro censu melioramento inde exeuntibus detis vos et vestri mihi et meis viginti septem libras monete perpetue regalium Maioricarum minutorum, in hunc modum videlicet: medietatem in festo Sancti Iohannis mensis iunii et alteram medietatem in festo Natalis Domini proxime venturis et abinde annis singulis in eisdem terminis sive festis [...]

Et pro hiis sic complendis et firmiter attendendis obligo vobis et vestris omnia bona habita et habenda ubique.

Pro intrata vero huius stabilimenti confiteor et recognosco me a vobis numerando habuisse et recepisse centum libras monete perpetue regalium Maioricarum minutorum $[\ldots]$

Ad haec, ego Guillermus Ros predictus, recipiens et acceptans a vobis, dicta Benencasa, dictum stabilimentum sub modis formis et conditionibus predictis, et promitto et convenio vobis et vestris dictum censum solvere et omnia alia et singula supradicta a me pertinentia attendere et complere sub bonorum meorum omnium obligatione.

Actum est hoc, in civitate Maioricarum, VII idus ianuarii anno Domini millesimo CCCXLIIII.

Signum Benencasa. Signum Guillermi Ros predictorum, qui haec laudamus et firmamus.

Signum Bertrandi Rubei, procuratoris predicti domini regis, qui haec firmo, salvo iure ipsius domini in omnibus et per omnia.

Testes: Francischus Oliverii curritor, G. Morro et Angelinus Dulcieti.

1353, septiembre, 16 .

Guillem Canterel paga un censo por unas casas cercanas al "Hort del Rei", en la "Ciutat de Mallorques".

ARM. ECR - 379, f. 7 v.

Margarita, mujer de Ferrarius Ses Eres establece a Guillermo Canterel [sic], civi Maioricarum, "quasdam domos quas habeo et possideo intus civitatem 
Maioricarum, prope hortum Domini Regis, in portione quae fuit nobilis domini Nunonis Sancii, quae nunc est domini regis.

Et affrontantur, ex una parte, cum domibus den Passamar et, ex alia parte, cum domibus Simonis Abennasser, et, ex alia parte, cum domibus de Na Spaera et, ex alia parte, cum via publica.

Deberá pagar 80 sueldos censuales cada año en la fiesta de Santa Maria de septiembre.

Testigos: Romeu Garcia, Ramon de Palomer, G. Servent.

1354, septiembre, 12 .

Guillem Canterell, brujulero y ciudadano de Mallorca, pagará un censo por un huerto en los aledaños de la "Ciutat de Mallorques".

ARM. ECR 381, f. 81.

"Pere de Cardona, civis Maioricarum, stabilio vobis Guillelmo Canterelli, bruxulerio et civi Maioricarum, licet absenti tanquam presenti, hortum in partita den Nuño Sanç, in orta civitatis". Linda con los huertos de Bernat Mayol, con camino público, con huerto den Planes y con huerto de Nicolau Mestre. Deberá pagar un censo anual, por Navidad, de 12 sueldos.

Testigos: Pere de Casanova, Miquel Aragonès, Joan de Claramunt.

6

1362, enero, 24.

Mención del albergue del brujulero Guillem Cantarell establecido en el doc. 4.

ARM. ECR-428, f. 230 v

Dulcia, uxor Arnaldi Xaverini, civis Maioricarum quondam et Nicolaus Xaverini, hijo suyo, venden a Jaume Diego, lanero y ciudadano, "quasdam domos quas habemus et possidemus intus civitatem Maioricarum, prope hortum Domini Regis... affrontantur dicte domus, ex una parte, cum domibus den Marcet et, ex alia parte, cum domibus Michaelis Cambi et, ex alia parte, cum domibus Guillermi Cantarell, buxolerii, et, ex alia parte, cum via publica". Precio: 9 libras. 
1402, agosto, 23.

Establecimiento de un albergue en la calle de Bonaire por Margarita, hija del brujulero Guillem Soler.

ARM. ECR 407, f. 387.

Nos, Stefanus Loreti et eius uxor Margarita, filia Guillermi Solerii, buxolerii, civis Maioricarum quondam, et domine Francesquine coniugum defunctorum, quae Francesquina soror fuit et heres abintestato domine Stefanie, uxor Iacobi Carreres quondam, gratis dono, stabilio et in enfiteusim perpetuam concedo vobis Iohanni Dominici, tintorerio, [...] quasdam domos meas situatas intus civitatem Maioricarum, in vico de Bonayra [...] affrontantur dicte domus, ex una parte, cum muro civitatis et, ex alia, cum hospitio quod fuit Iacobi Paratge et nunc est Guillermi Boneti, tintorerii, et, ex alia, cum hospitio quod fuit den Babiloni et nunc est Thome Boneti et, ex alia parte, cum dicto vico de Bonayra [...]

Testes: P. Paschasii, curritor, Andreas Durandi et P. Vitalis, paratores, cives Maioricarum*.

* Cancelado el 31-8-1408. El 29-8-1408 [sic] se concede a Geraldo Collet, textori, por 32 sueldos anuales.

1437, junio, 18.

El brujulero Gabriel Vallseca posee ciertos derechos sobre una esclava semanera.

ARM. Prot. A-86, ff. 73-73v.

Sit omnibus notum quod ego Gabriel Vallsecha, buxolerius, civis Maioricarum, gratis et scienter confiteor et recognosco vobis, Iacobo Massanet, civi Maioricarum, quod dedistis et solvistis mihi et ego a vobis habui et recepi numerando, ex una parte, tresdecim libras re.ma.mi. quas ego vobis tradideram, ex eo quia, pro illis, vos debebatis mihi vendere certum ius, vobis in et supra quadam serva pertinens, usque ad dictas terdecim libras pro quibus mihi debebat facere septimanam, quam tamen servam vos etiam postea vendidistis alteri, nulla facta mencione de dictis terdecim libris, ob quod fuistis, ad illam restitutionem mihi 
fiendam, condempnatus per sententiam locumtenentis gubernatoris. Et, ex alia parte, dedistis et solvistis mihi tres libras et duodecim solidos, occasione missionum per me factarum occasione dicte sententie, $[\ldots]$

Actum est hoc in civitate Maioricarum, die martis, decima octava mensis iunii, anno a Nativitate Domini millesimo CCCC tricesimo septimo.

Signum mei, Gabrielis Valseca predicti, qui hec laudo et firmo.

Testes inde sunt: Ludovicus Jover, ballistarius, et Laurentius Camps, sartor, cives Maioricarum.

1438, noviembre, 24 .

Gabriel Valseca "mestre de cartes de navegar" como novel poblador recibe del "Gran i General Consell" inmunidad de cargas por diez años.

ARM. AH-3 [1438-1440], Actes del Gran i General Concell, f. 19.

Com es acostumade en aquesta ciutat per vosaltres, honorables jurats e General Concell l'any present [1438], que, a quade un poblador novell de la vostre ylla, donau e atorgau inmunitat per deu anys de prevere, per tant, $a b$ aquelles humils suplicacions que fer, ne dir se pot, suplique en Gabriel Vallsecha, mestra de cartes de navagar, que, a ell la dita franquesa vullau atorgar, la qual cosa reputerà a gràcia singular.

Postmodum vero die lune mensis et anni predictorum, honorabiles Ioannes de Turricella domicellus, Obertinus de Dameto, Ferdinandus Valentini, Petrus Çavila et Iulianus Quintana, quinque ex dictis honorabilibus iuratis dicte universitatis Maioricarum, vigore potestatis eidem per dictum Generalem Consilium die proxime lapsa attribute, concorditer concesserunt dictam gratiam et libertatem dicto Gabrieli Vallsecha, iuxta supplicata.

1443, marzo, 15.

Gabriel Valseca brujulero adquirió una casa, en Mallorca del mercader florentino Giovanni de Manzoli.

ARM. Prot. A-88, f. $192 v-193$. 
Sit omnibus notum quod ego, Gabriel de Vallseca, buxolerius, civis Maiorcarum, gratis et scienter, confiteor et recognosco me debere vobis, venerabili Matheo Vaquer, civi Maioricarum presenti, quadringentas triginta libras, decem solidos regalium Maioricarum minutorum, quas mihi gratiose mutuastis et numerando ego a vobis habuit et recepi, seu verius illas de voluntate et mandato meis tradidistis et solvistis lohanni de Manzoli, mercatori florentino, pro nunc in presenti civitate Maioricarum residenti, procuratori venerabilis Benedicti de Iohanni Manzoli, mercatoris civitatis Florencie, fratris et heredis Nicholai de Iohanni, mercatoris quondam, in civitate Maioricarum dum vivebat degentis, in solutum consimilis pecuniae quantitatis in qua ego sibi tenebar, pro pretio cuiusdam hospitii, hereditatis dicti quondam Nicholai de Iohanni, situati intus civitatem Maioricarum, iuxta plateam del Born, per ipsum mihi venditi, et, ab ipso Nicholai de Iohanni venditori, obtinuistis cessionem pro securitate vestra et dicte quantitatis pecunie, per vos sibi pro me, ex causa predicte sulutionis, prout constat per instrumentum inde factum in scribania instrumentorum domini regis hodierna die, paulo ante facto.

Cum cessione per vos obtente, nolo, per huiusmodi instrumentum, in aliquo derogari seu novationem fieri, quin inmo in suo robore perduret et ipsis iuribus et actionibus vobis cessis uti et gaudere valeatis, quotiescumque vobis fuerit opus et quousque ipsa quantitas fuerit, per me, vobis, integriter restituta et soluta.

Unde renuntiando exceptioni pecunie predicte, ex causis predictis per me vobis non debite et in solutionem dicti pretii prefati hospitii non converso et doli, promitto dictas CCCC XXX libras, X solidos, vobis dicto Mateo persolvere in hunc qui sequitur modum, videlicet: hinc et per totum mensem aprilis proxime venturum, ducentas octuaginta libras decem solidos, et, pro residuis centum quinquaginta libras, promitto facere vobis venditionem de duodecim libris censualibus super certo alodio et omnibus bonis meis onerandis, omni dilatione, contradictione et exceptione remotis.

Super quibus volo credi vobis et vestris solo plani et simplici verbo, absque testibus et iuramento et nullo alio probarum genere requisito. Obligando inde vobis, specialiter et expresse ac iure proprii pignoris et specialis obligationis, dictum hospitium per me emptum, a dicto Iohanni de Nicolo, quod constituo vestro nomine precario tenere et possidere et illud non vendere seu alienare aut alicui obligare in preiudicium huiusmodi vestre obligationis et quousque in dicta pecunie quantitate fuerit per me vobis integriter satisfactum [...]

Actum est hoc in civitate Maioricarum, die veneris XV mensis martii, anno a Nativitate Domini millesimo CCCC quadragesimo tertio.

Signum mei, Gabrielis de Vallseca predicti, qui haec laudo et firmo.

Testes inde sunt: Iohannes de Nicolò de Manzoli, mercator Florentie et Petrus Manzoli, mercator, civis Maioricarum. 
1446.

El brujulero Gabriel Soler habita en la parroquia de Santa Cruz, en la manzana del mercader Pere Pardo.

ARM. AH 6731 s.f.

Talla de las parroquias de la Ciudad de Mallorca del año 1446 Parroquia de Santa Creu. Illa den Pere Pardo [...] Gabriel Soler, buxoler,... trenta sous.

1463, diciembre, 16 .

El brujulero Gabriel Soler comparece como testigo en una transacción mercantil.

ARM. Prot. J. Falcó, núm. 4743, f. 64 .

Bernat Verdera, mercader de Mallorca, recibe diversas alhajas de plata, oro, diamantes y coral del peraire Gerald Cerdà, tras sentencia judicial.

Testes: honorabilis Anthonius Sparech, iurisperitus, et Gabriel Soler, buxolerius, et Antonius Bertran, caput excubiarum.

1467, noviembre, 13.

Gabriel Soler, maestro de cartas de navegar, testifica en un contrato portuario.

ARM. Prot. C-112, f. 99v.

Ego Andreas de li Strossi, mercator civitatis Maioricarum, gratis etc. vendo vobis, Alfonso Portogues, marinerio eiusdem civitatis Maioricarum, genero Blasii Romei, fusterii Maioricarum presenti etc unum quarterium cuiusdam barche riparie vocate "L'artibanch", una cum quarta parte omnis eius exarciarum et arreus etiam 
cum patronia ipsius barche, pretio videlicet viginti sex librarum, tresdecim solidos, septem denariorum [...]

Testes Gabriel Soler, magister cartarum naviguandi, civis Maioricarum, et Iohannes Pages, oriundus ville Perpiniani.

1451, marzo, 13.

Antoni Bramona, brujulero sarraceno, alquila un local en el Borne de la "Ciutat de Mallorques".

ARM. Prot. Notario Bernat Contestí, C-105, f. 73v.

Nos Anneta, uxor venerabilis Petri de Brulio, mercatoris, civis Maioricarum, nunc a presenti regno Maioricarum absenti, et Miquael de Brulio, filius dictorum coniugum, gratis etc. locamus tibi Antonio Bramona, nationis sarracenorum, buxolerio eiusdem civitatis Maioricarum, presenti, etc. quandam partem cuiusdam hospitii mei, dicte Annete, infra menia dicte civitatis Maioricarum constructi, satis prope Burnum ipsius civitatis, videlicet totum patuum dicti hospitii inferius et omne superius, dempta tamen intrata dicti hospitii a purtali vici den Tome Thomas usque ad pedem scale ipsius hospitii [...] ad unum annum, a die presenti in antea continue numerandum, loguerio vel mercede quatuor librarum regalium Maioricarum minutorum, quolibet anno, [...].

Testes: Mateus Stephani et Georgius Munarii, habitatores parrochie Andragii.

1454, enero, 14 .

Ubicación del albergue del brujulero Rafel Monells en la calle del Mar.

ARM. Liber notularum curie baiulie AH 5932 [años 1452-1454] s.f.

Al molt honorable en Jacme Rossinyol, batle de Mallorques, o a son lochtinent, de nos, en Nicholau de Pachs e en Joan de Bas, consols l'any present dels actes e negocis mercantils de la Ciutat e Regne de Mallorques, saluts ab crexement de tota honor. 
Com aprés diverses manaments per part nostre, a instància den Yego de Jahent, sastre de Mallorques, fets a na Ysabel Paris, de pagar al dit Enyego, onze liures, deu sous, a ell per ella degudas, per rahó d'un drap blanch que lo dit Enyego li ha venut, no havent la dita Ysabel bens mobles per pagar aquell, segons ha jurat als sants Quatre Evangelis per ella corporalment tocats, havia donades en nostra cort e assignades unes sues cases e algorfa o soler de sobre, los quals ha e posseeix en la parròquia de Sancta Creu, en lo carrer de la Mar, fora lo portal vulgarment dit den Segarra, e les quals eren den Johan Marçol, pintor de Mallorques e de na Maria, amiga sua, e aprés den Rafel Monells, buxoler de la dita Ciutat, qui aquellas ha comprades dels dits Johan Marçol e dita Maria, segons que de la compra del dit Rafel Monells appar, per carta d'aquen, feta en poder del discret en Genís Mianes, notari de Mallorques, sots dimarts, a dos del mes de Març de l'any de la Nativitat de Nostre Senyor mil CCCC quoranta cinch, a subastar e a vendre, per pagar e satisfer al dit Enyego les dites onze liures ensemps ab les missions.

E açò per spay de vuit dies, com haia maior temps, ço es de quatre mesos renunciant en aquells pactats $[\ldots]$

E les quals dites cases ab la dita algorfa son tengudes sots alou e dreta senyoría dels honrats en Francesch Asselm e den Thomàs Serra, ciutedans de Mallorques [...].

E affronten les dites cases ab la dita algorfa, de una part, ab lo carrer de la Mar, e, de la altre part, ab un carreró per lo qual se entra en la Riera de la present Ciutat, e, de la altre part, ab lo mur de la Riera e, de la altre part, ab les cases de $\mathrm{Na}$ Graciosa, muler den Gonsalvo Ferrando, portoguès, tender quondam.

On, com la exequció de les dites cases e algorfa ordinariament pertangue a vos e a vostra jurisdictió e cort, per tant, observant d'aquen lo Capitol de Consolat sobre açò fet e ordenat, ab tenor de les presents, instant e requirent lo dit Enyego de Jayent, remetem a vos e a vostre ordinari examen e de la dita vostre cort, la exequció d'aquellas.

Requerint vos d'aquen, en subsidi de justicia, de part del senyor rey e de la nostra, exhortant e pregant que deduhits los drets de la dita vostra cort e los treballs de vostre discret notari e scrivà, fets en la distracció e exequució d'aquelles, lo residuum del preu d'aquells remetats a nos e a la nostra cort, per ço que de aquell residuum puscam contentar satisfer e pagar al dit Enyego de Jayent, en les dites onze liures, deu sous e messions fetes, iustitia migensant.

Datum Maioricis, die XIIII mensis ianuarii, anno a Nativitate Dni. millessimo quadringentesimo quinquagesimo secundo.

[...] Et cum nullus repertus fuisset qui in dictis domibus et algurfa tantum daret quantum domina Elena, uxor Amorós, hostalerii, que in illis dixit de pretio viginti libras, [...] pro tanto, Georgius de Sancto Iohanne, miles, baiulus Maioricarum [...] dedit baculum sive astam fischalem de predictis domibus et algurfa dicte domine Elene $[. .$.$] .$ 
1463, julio, 15.

Pere Rossell, brujulero, adquiere ciertas telas.

ARM. Prot. M-412, f. 63.

Ego Petrus Rossell, buxolerius, civis Maioricarum, gratis confiteor debere vobis venerabili Thome Cirera, mercatori Maioricarum VIII 11., XV ss. re. Ma.mi. pretio quorum a vobis emi quendam pannum album setzè lane de la terra pro induendo me et persona mea...

Est tamen certum quod vos tenetis in pignus un cubertor listat ab la cuberta de cotonina listada de listes vermelles e blanques ab barres blanques e I sabana de tela blanca e un tapaportes de dues figures.

Testes: Bartomeu Rovellat, curritor auris, civis, et Iohannes Valls.

1474, junio, 7 .

El maestro brujulero Pere Rossell adquiere una provisión de aceite.

AHM. Prot. A-100, ff. $30 v-31$.

Ego magister Petrus Rossell, buxolerius de Maioricis, gratis confiteor etc. me debere vobis, honorabili Anthonine, uxori honorabilis Petri Vivot, domicelli de Maioricis, licet absenti, quinque libras re.Ma.mi., quarum pretio, ego, a vobis habuit et recepi numerando et vos mihi tradidisti, viginti quinque quartanos olei, ad rationem videlicet quatuor solidorum pro quolibet quartano.

Unde rerum etc promitto vobis illas dare et volvere per totum mensem augusti proximi $[\ldots]$

Testes: venerabilis Antonius Axertell, civis Maioricarum, et Miquel Colomar, civis Valencie. 
1500 , septiembre, 7 .

Se da por fallecido al brujulero Jaume Bertran.

ARM. Prot. Nic. Costa, num. 4494, f. 45.

Ego Iohannotus Nicholaus Bertran, filius Iacobi Bertran buxolerii quondam, sciens et attendens super questionibus et differentiis inter me, dictum Iohannotum, Nicholaum, ex una parte, et dominam Francinam, uxorem Petri Camps, partibus ex alia, vertentibus, pretextu et occassione videlicet quia ego habere pretendebam, a dicta Francina, uti substituta herede sororibus meis predefunctis per prefatum patrem meum, legitimam seu illius suplementum ex dictis bonis, parte ex una, et, insuper, a dicta Francina habere pretendebam medietatem portionis bonorum dicti patris mei pertinentis Margarite quondam sororis mee, novissime in pubertate deffuncte, [...] pro evitandis expensis et dissensionibus [...].

1513, enero, 12.

Joan Salvat "illuminator" coloca a su hijo Joan Benet Salvat como aprendiz de orfebre.

ARM. Prot. M-280, ff. $54 v-55$

Ego, Iohannes Salvat, illuminator, gratis mitto et affirmo vobiscum, Iohanne Sant Just, aurifabro Maioricarum, presenti, Iohannem Benedictum Salvat, filium meum, etatis XVI annorum vel circa, causa adiscendi officium vestrum ad perfectionem et serviendi vobis etc., ad tempus novem annorum, a die presenti ab inde numerandorum, cum iam steterit cum aliis magistris per multos annos, promittens quod dictus filius meus non aufugiet, nec damnum aut furtum faciet vobis, quod si faceret, promitto etc., largo modo vosque possitis etc.

Vos, vero, teneamini docere etc., providere in cibo et potu etc., et, pro vestitu, dare eidem anno quolibet, quatuor libras, pro quibus obligo personaliter et bona largo modo.

Ad haec, ego, dictus Iohannes Sant Just, presens, etc., promitto etc., ut supra, obligo personaliter et bona. 
Testes: Antonius Mut, scriptor, et Martinus Amorós, parrochie de Muro, firmam amborum predictorum [...].

1520, agosto, 3 .

El brujulero Joan Salvat se compromete al pago de una imposición.

ARM. Prot. R-587, ad diem.

Nos, Iohannes Salvat, buxoler, Catherina Blanqua, uxor Pontii Sala quondam, Hieronyma, sponsa Iohannis Salvat buxoler, gratis, sine preiudicio primarum obligarionum, nos obligamus, simul et in solidum, solvere vobis magnifico Bernardo de Juny et Georgio de Santacilia, clavariis universitatis Maioricarum, absentibus, et successoribus vestris in dicto officio* monete Maioricarum, restantes ex pretiis impositionis encantuum[?] anni videlicet MDXXI et MDXX, per dictum Pontium Sala quondam, textorem, quas solvere promittimus sine omni dilatione $[\ldots]$.

Testes: Gabriel Domenech, blanquerius, et Antonius Mas, calsaterius.

* Sigue un blanco.

1498, septiembre, 5 .

El portugués Álvaro Viera se compromete a trabajar en el taller de Bartomeu Oliva, pintor.

ARM. Prot. R.-770, f. 42

Ego Alvarus Viera de Lisboa, residens in presenti civitate Maioricarum, gratis et ad tempus unius anni a die presenti in antea computandi, mitto personam meam vobiscum Bartholomeo Olives, pictori, civi Maioricarum, causa serviendi vobis in officio de pintor.

Promitto vobis quod, durante dicto tempore, non recedam a dicto vestro servitio, quod, si fecero, non possim stare hic Maioricis cum alio magistro pinctor. 
Attamen, vos teneamini me tenere sanum et aegrum, in vestro cibo et potu et in fine dicti temporis dare et tradere octo ducatos auri in auro boni et iusti ponderis.

Hoc tamen acto et convento inter me et vos quod, si casus evenerit ego me voluere conferre a dicto vestro servitio infra annum, teneamini solvere mihi omne tempus quod vobis serviero, numerando prorrata de dicto pretio.

Ad haec, ego, Bartholomeus Olives antedictus, hiis presens et vos dictum Alvarum Viera in servitium meum, sub pactu et conditionibus supradictis, acceptans, promitto, finito tempore predicto, dare et tradere vobis dictos octo ducatos seu, si ante annum vos conferebatis, solvere id quod erit $[\ldots]$

Testes: Iohannes Babtista Rodi et Bartolomeus Alberto, mercatores ianuenses, pro nunc residentes in presente civitate Maioricarum, in quorum presentia firmamus.

1506, octubre, 26.

El pintor Bartomeu Olives decora el catafalco del funeral de Felipe el Hermoso.

ARM. RP 3665 Dades, ad diem.

Item paguí en Bartomeu Olives, per LII senyals reyals patits, qui estaven meses als ciris qui estaven al entorn de la tomba [del rei Felip de Castella e primogénit d'Aragó], a raó de VI d. la pessa, ...1 liura, V sous VI diners.

Mes li paguí, per XIII senyals reyals grans, qui staven sobre lo capell ardent e sobre la tomba e posats sobre los draps qui estaven al entorn del pati del castell, a raó de $\mathrm{V}$ sous la pessa... III liures, $\mathrm{V}$ sous.

Item mes li paguí, per VIII senyals reyals per metre al entorn del pavelló del capell ardent... XVI sous.

1514 , enero, 14

Últimos sacramentos de Bartomeu Olive, pintor.

ACM. L de sacristia 1514 s.f. 
A XVIIII de janer combregà mestre Bartomeu Olive, pintor. Anaren-hi deu ciris... III sous, IIIII diners.

1526, diciembre, 31 .

Bartomeu Oliva, pintor, y Joanot Salvà, orfebre, hacen de consuno una compra de trigo al mercader Galceran Berard.

ARM. Prot. R-778, f. 161 v

Nos, Bartolomeus Oliva, pictor, et Ioannotus Salvà, aurifaber, cives Maioricarum, gratis etc, confitemur et recognoscimus debere vobis honorabili Galcerando Barard, mercatori, civi Maioricarum, VI libras, XVI solidos re.Ma.mi., pretio quarum a vobis mercantiliter emi, habui et recepi, ego, dictus Oliva, et de voluntate mei dicti Salvà dicto Oliva tradidistis, octo quarterias frumenti alieni boni, nitidi et purgati.

Ideo promittimus uterque nostrum in solidare et solvere vobis in Festo Omnium Santorum proxime venturo [...]

Testes: Michael Eleu, mercator, et Iohannes Palagrí, parator, cives Maioricarum [...]

1530 , abril, 16.

Un brujulero trabaja en la decoración de Pascua en la Seo de Mallorca.

ACM. L. de sacristia 1515 , s.f.

Item a XVI d'abril [1530], nit de Pasco, doní an el buxoler, per a pintar lo pregamí que possan al ciri pasqual un ducat... I Il. XII ss.

1532, junio, 27. 
Bartomeu Olives, pintor, adquiere un gran lote de pergaminos.

ARM. Prot. Pere Arques, Man. 1526-1555, f. 210v.

Ego Bartolomeus Olives, pictor Maioricarum, gratis confiteor et recognosco debere vobis, honorabili Iohanni Benedicto, mercatori Maioricarum, presenti, viginti quatuor libras re Ma. mi., pretio quarum a vobis mercantiliter emi, habui et recepi triginta duodenas pergamenorum sive pregamins, tales quales sunt et de quorum bonitate ego valde concensor, ad rationem sexdecim solidos pro qualibet duodena, et volo ad opus officii mei pictoris.

Ideo renuntio etc. et promitto ipsas dare et solvere vobis in hunc sequentem modum, videlicet, a die presente et infrascripta ad duos menses proxime venturos medietatem, et, lapsis ipsis duobus mensibus ad alios duos menses infra sequentes, restantem quantitatem $[\ldots]$ Maioricarum.

Testes: Gaspar Rabasa, calsaterius, et Iacobus Aguiló cursor auris

1532, octubre, 12 .

Bartomeu Olives, pintor, poseedor de un huerto.

ARM. Prot. C-311, f. 13 v.

Nos Bartolomeus Oliva, pictor, et Francina, eius uxor, [...] promittimus [....] vobis domine Caterine, uxori relicte honorabilis Ioannis Ortolà quondam et vestris, quod quotiescumque damnum aliquod vobis evenerit causa et occasione arborum per me seminatorum in quodam horto per me empto, contiguo quibusdam domibus et horto vestris dicte domine Ortolane in quadam fonte per vos facta in horto vestro, illud damnum vobis exsolvere [...] et promitto non seminare in dicto meo horto ladoner ni figuera $[\ldots]$.

1538, marzo, 13.

Bartomeu Olives, pintor, establece una casa en la calle del Mar de la "Ciutat de Mallorques". 
ARM. Prot. Ll-96, ad diem.

Noverint universi quod nos, Bartolomeus Olives, pictor Maioricarum, et Francina eius uxor, gratis et scienter, damus, stabilimus et in emphiteusim perpetuam concedimus et tradimus vobis Guillermo Andreu, bombarderio Maioricarum, presenti et domine Apalonie uxori vestre, absenti ut presenti, et vestris, quasdam domos nostras, algurfam et botigiam situatas intus civitatem Maioricarum, in parrochia Sancte Crucis, in vico Maris, ante almudinum presentis civitatis, confrontatas, ex una parte, cum via publica, et, ex alia parte, cum domibus domine Ysabelis Aragonesa, et, ex alia parte, cum rieria presentis civitatis, et, ex alia parte, cum aliis domibus nostris [...]

Sub tamen tali pacto et conditione quod pro censu $[\ldots]$ detis $[\ldots]$ anno quolibet $[\ldots]$ in festo Santi Gregorii sex libras monete Maioricarum [...]

Actum est hoc, in civitate Maioricarum, die tredecima mensis martii, anno a Nativitate Domini millesimo quingentesimo trigesimo octavo.

Signa nostrum Bartolomei Olives et Francine, stabilitorum. Signa nostrum Guillermi Andreu et Apalonie, coniugum acquisitorum predictorum, qui haec laudamus et concedimus.

Testes: Ioannotus Genovard, studens, et Franciscus Bartomeu, textor lini Maioricarum [...].

1546, abril, 18.

Bartomeu Olives, iluminador de cartas de navegar, contrae matrimonio y recibe la dote.

ARM. Prot. C-295, ff. 230-230v.

Ego Bartholomeus Olives, illuminator cartarum navegandi, sciens et attendens me contraxisse matrimonium vobiscum, domina Margarita, uxore mea, filia Benedicti Panadés, paratoris Maioricarum, et domine Caterine, coniugum, et dictus Benedictus Panadés, pater vester, constituisse in dotem et nomine dotis vestre promississe centum libras monete Maioricarum solvendas ad meas voluntates illasque, a dicto patre vestro, habuisse et recepisse omni modo voluntati et nullo vobis fecisse seu firmasse sponsalicium nostrum, ideo, volens bonam vobis agnoscere fidem, gratis etc., confiteor et recognosco habuisse et recepisse dictam dotem omnimode voluntati mee et facio vobis augmentum de quarta parte dicte dotis. 
Et sic sunt, inter dotem et augmentum, centum et viginti quinque libre quamquidem dotem etc. $[\ldots]$

Testes: Laurentius Ferrer et Matheus Lorens, vidrierii Maioricarum.

1563, junio, 14 .

Comparecencia de Francina Oliva, viuda del pintor Bartomeu Oliva.

ARM. Prot. V-434, f. 170 v.

Ego Francina Oliva, vidua, uxor relicta honorabilis quondam Bartolomei Oliva, pictoris, gratis etc. promitto vobis, magistro Bartolomeo Ferra, ferrifabro presenti, quod ego faciam et firmabo vobis instrumentum venditionis seu stabilimenti illarum domorum de quibus vobis firmavit instrumentum venditionis magister Raphael Oliver, lapiscida [...]

Testes: honorabiles Ioannotus Salvat, aurifaber, et Lucas Mager, lignifaber.

1609, julio, 28.

Testamento de Vicens Prunes cosmografo, vecino de la parroquia de Santa Eulalia.

ARM. Prot. C-786 s.f.

In Dei nomine et eius divina gratia.

Cum scriptum fuit per prophetam dicentem: Dispone domui tuae quia morieris et non vives, idcirco, ego, Vincentius Prunes, cosmographus, infirmitate corporali in lecto detentus, de qua mori timeo, in meo tamen pleno sensu, memoria integra et firma loquela existens, volens de bonis mihi adeo collatis disponere, hoc meum facio, condo et ordino testamentum, in et cum quo meos pono et eligo manumissores et huius mee voluntatis exequutores, scilicet honorabilem dominam Peretam Prunes et Puig, charissimam uxorem meam, et honorabilem Guillermum Amengual, afinem meum, et utrumque illorum insolidum quos rogo, prout carius possum eisque plenam do et confero potestatem quod, si me mori contingerit antequam aliud mihi liceat condere testamentum, ipsi, de bonis meis, hanc compleant 
et exequantur ultimam voluntatem, prout inferius descriptum invenerint et per me ordinatum.

Et, in primis et ante omnia, commendans animam meam in manus Domini Nostri lesuchristi, eligo sepulturam cadaveri meo faciendam in ecclesia parrochiali Sancte Eulalie, in vase meorum predecessorum, quam fieri volo ad cognitionem meorum manumissorum.

Deinde, ut misericors Deus veniam largire dignetur omnibus peccatis meis, facio pia legata sequentia: Primo lego reverendo rectori, cuius parrochianus fuero tempore obitus mei, pro iure suo parrochiali, quinque solidos. Item lego et, incontinenti morte mea sequuta vel eo citius quo fieri poterit, celebrari mando quindecim missas de requiem, pro una vice tantum, summissa voce, in altaribus privilegiatis, dictis meis manumissoribus benevisis, pro quibus lego charitatem assuetam.

Item lego omni parte hereditate et legitima Matheo Prunes filio meo absenti a presenti Maioricarum Regno, Petro Ioanni Prunes, Marie Prunes domicelle et Iohanni Baptiste Prunes, filiis meis et unicuique illorum, singulos quinque solidos, in quibus illos et quemlibet illorum mihi heredes particulares instituo.

Item, lego, omnibus liberis meis amodo nascituris postumis et medio tempore natis, singulos quinque solidos, in quibus illos et quemlibet illorum mihi heredes particulares instituo.

Solutis autem et completis omnibus et singulis iniuriis et debitis meis sive torts, quae apparuerint tempore obitus mei, plenarie restitutis, quae solvi et satisfieri volo, iubeo et mando plane et sine strepitu nec figura iudicii sed sola facti veritati asserta, malittis et diffugiis retro eiectis, inomnibus autem aliis bonis mei mobilibus et inmobilibus $[\ldots]$ instituo et facio heredem meam universalem predictam dominam Paretam, uxorem meam charissimam [...]

Quod fuit actum, in presenti civitate Maioricarum, die vigessima octava mensis iulii, anno a Nativitate Domini millessimo sexcentesimo nono.

Signum meum Vicentii Prunes, cosmographi $[\ldots]$

Testes huius testamenti, proprio ore ipsius testatoris vocati et rogati, sunt: honorabilis Petrus Ripoll, nauta; Christophorus Barceló, nauta; Iacobus Rotger, nauta; Damianus Noguera, lodicarius; Michael Bertran, lodicarius dis; Franciscus Stada, studens, et venerabilis Michael Bastard, beneficiatus in ecclesia alme Sedis Maioricarum, et ego, notarius infrascriptus, qui rogatus et requisitus dictum testamentum recepi.

Obiit, dictus testator, die quinta augusti millesimi sexcentessimi noni, ante ortum solis, in aurora. Cuius anima in pace requiescat. Amen. 


\section{RÉSUMÉ}

Le port de Ciutat de Mallorques fût très important pour l'histoire de la cartographie médiévale, parce-qu'un certain nombre de dessinateurs et copistes de cartes s'établirent là au commencement du XIV siècle, en raison de l'ouverture de la route atlantique. Maintenant, ces cartes se trouvent dispersées en diferents musées par tout le monde, Les auteurs de ces cartes étaient connus et datés jusqu'aujourd'hui à travers ses ouvrages. Dans cet article nous est donné un premier registre des maîtres de cartes de naviguer que travaillèrent au port de Majorque, provenant de sources locales, notariales et administratives, que nous permettent d'eclircir leurs noms, leur date de naissance leur Sitz im Leben et leurs méthodes de travail, peu après débordés et dépassés par la presse moderne.

\section{SUMMARY}

In medieval times, with the opening of the Atlantic trading routes at the beginning of the 14th century, the port of the Ciutat de Mallorques became important as the base of a gathering of both cartographers and copiers of maps. Today these maps are dispersed in museums throughout the world. Until the present day, these early map makers were known only through their works. Presented here is the first register of the "maestros" of navigational charts who worked in the "port of Mallorca". The documentation is taken from local notarial and administrative sources, all of which help the better clarification of their names, birthplaces, their Sitz im Leben and their methods of production, which were later surpassed and improved by the advent of the modern printing press. 